\title{
Marie-Hélène Parizeau et Soheil Kash (dir.), La Société robotisée. Enjeux éthiques et politiques
} Québec, Presses de l'Université Laval (Bioéthique critique), 2019

\section{Guillaume Carnino}

\section{(2) OpenEdition}

\section{Journals}

Édition électronique

URL : http://journals.openedition.org/artefact/6167

DOI : 10.4000/artefact.6167

ISSN : 2606-9245

\section{Éditeur :}

Association Artefact. Techniques histoire et sciences humaines, Presses universitaires du Midi

\section{Édition imprimée}

Date de publication : 15 juillet 2020

Pagination : 378-381

ISBN : 978-2-8107-0691-4

ISSN : 2273-0753

\section{Référence électronique}

Guillaume Carnino, «Marie-Hélène Parizeau et Soheil Kash (dir.), La Société robotisée. Enjeux éthiques et politiques ", Artefact [En ligne], 12 | 2020, mis en ligne le 21 décembre 2020, consulté le 23 décembre 2020. URL : http://journals.openedition.org/artefact/6167 ; DOI : https://doi.org/10.4000/artefact. 6167

Ce document a été généré automatiquement le 23 décembre 2020.

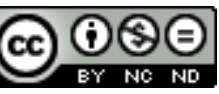

Artefact, Techniques, histoire et sciences humaines est mise à disposition selon les termes de la Licence Creative Commons Attribution - Pas d'Utilisation Commerciale - Pas de Modification 4.0 International. 


\section{Marie-Hélène Parizeau et Soheil Kash (dir.), La Société robotisée. Enjeux éthiques et politiques}

Québec, Presses de l'Université Laval (Bioéthique critique), 2019

\section{Guillaume Carnino}

\section{RÉFÉRENCE}

Marie-Hélène Parizeau et Soheil Kash (dir.), La Société robotisée. Enjeux éthiques

et politiques, Québec, Presses de l'Université Laval (Bioéthique critique), 2019, 374 pages.

1 La société robotisée. Enjeux éthiques et politiques est un ouvrage collectif constitué de contributions présentées au colloque international et interdisciplinaire «Robots et sociétés: quelles transformations? Quelles régulations?», qui s'est déroulé à l'Université Laval à Québec en mars 2017. Les vingt et une contributions réunies dans l'ouvrage sont majoritairement le fait de philosophes, auxquels se mêlent des acteurs aux prises avec le monde technologique contemporain (un juriste, un physicien, une pédiatre, un gériatre, un informaticien et quelques spécialistes de l'éducation).

2 Après une introduction où Marie-Hélène Parizeau et Soheil Kash évoquent les vicissitudes du terme de robot et discutent des implications du phénomène robotique au niveau du travail notamment, le chapitre d'ouverture est une contribution du philosophe Jean-Michel Besnier. Il y défend la thèse selon laquelle le robot est aujourd'hui l'exutoire des aspirations et utopies contemporaines, symptôme d'un malêtre civilisationnel et d'une fatigue ayant pris des dimensions anthropologiques.

Le cœur de l'ouvrage s'organise en deux parties, la première, intitulée «Le robot comme objet technique: enjeux philosophiques et éthiques" regroupant neuf contributions, et la seconde, traitant des « Robots dans la société : enjeux éthiques et politiques ", douze. Les deux parties, tant en taille qu'en densité du contenu, s'avèrent assez inégales à la lecture : si tout colloque possède son lot d'interventions bien menées 
et documentées précisément, cohabitant bon an mal an avec des textes moins fouillés (ou simplement ennuyeux), la distinction semble ici d'un autre ordre, et relever spécifiquement de cette thématique qu'est la robotique.

4 Les deux parties s'opposent principalement sur le statut du « robot ». Dans la première, il s'agit surtout d'un objet d'investigation hypothétique : peut-on et faut-il étendre notre morale aux robots (Vanessa Nurock, Tomislav Bracanović) ? Comment anticiper les développements de la robotique du point de vue juridique (Charles-Étienne Daniel), politique (Hoda Nehmé), cognitif (Frédéric Dubois) ou sociopsychique (Alexandre Pitti)? Les deux dernières contributions de ce premier ensemble reviennent à des dimensions moins prospectives en retournant l'analyse, puisqu'il s'agit de saisir ce que la logique de la robotique fait au vivant - à travers la biologie de synthèse (Guillaume Pelletier) - et à l'être humain - pris dans un devenir-robot capitaliste (Chantale Pilon). La quasi-totalité de ces textes souffre du défaut de toute étude prospective: le fondement de ce genre théorique est bien souvent labile au point de faire douter de l'intérêt de consacrer du temps de réflexion à des phénomènes qui n'adviendront peutêtre jamais. Or, la robotique exacerbe ce risque, tant le domaine est saturé d'anticipations plus ou moins folkloriques, bien que tenaces historiquement. Le mythe de l'automatisation de l'activité humaine par les robots est si éculé qu'il en a acquis une histoire $^{1}$. On peut aussi se souvenir des déclarations tonitruantes d'experts économiques, de ténors médiatiques, d'instituts de prospective et de responsables politiques à la fin des années 2000, annonçant que les années 2010 seraient placées sous le signe du robot et qu'elles marqueraient l'explosion du marché de la robotique appliquée au quotidien. Si la chirurgie ou l'aviation ont en effet été impactées par les robots (dans une proportion qu'il conviendrait d'étudier au demeurant), la vie courante n'a guère été bouleversée, si l'on excepte l'émergence timide de quelques aspirateurs automatiques dans certains foyers. Ou plutôt, si des robots réels monitorent certains aspects de notre existence, il s'agit bien davantage d'algorithmes de masse, qui certes sont aussi le véhicule de nombreux mythes, mais qui ne correspondent en rien au stéréotype du robot humanoïde.

5 La seconde partie a le mérite de présenter des contributions majoritairement construites à partir de terrains précis. Du même coup, elle colle davantage à la réalité du monde contemporain et se perd moins dans des conjectures hasardeuses. On y découvre quelques conséquences, notamment stratégiques, de l'automatisation des armements - à commencer par les drones tueurs (John Finney). Dans une veine déflationniste bienvenue par rapport aux discours prospectivistes, Soheil Kash remarque que le drone tueur cohabite avec le kamikaze - deux extrêmes du rapport entre le soldat et son corps aboutissant à redéfinir la dialectique maître-esclave hégélienne. Interrogeant l'incitation économique à l'expérimentation in vivo que constitue l'introduction de robots dans de nombreux secteurs de la vie quotidienne (domicile, hôpital, école, etc.), Marie-Hélène Parizeau réinvestit la critique formulée par Günther Anders à l'égard du devenir machine de l'être humain. Plusieurs textes questionnent ensuite l'efficacité et la pertinence des robots en situation concrète, notamment pour les enfants et les personnes âgées. Dans le cadre du traitement des troubles du spectre autistique, Josée-Anne Gagnon critique le robot comme une entité non-relationnelle, tout comme Sophie Cloutier - à partir des travaux de Sherry Turkle oppose relation authentiquement réciproque (comme avec les animaux) et situation de contrôle (avec les machines) - cette dernière ne créant pas d'empathie et induisant un inconfort émotionnel face à toute altérité non maîtrisée. Raoul Kamga et Margarida 
Romero ouvrent la boîte noire des inégalités pédagogiques et économiques favorisées par un marché de la robotique gourmand en capitaux. Concernant les personnes âgées, Félix-Antoine Pageau observe des robots qui s'avèrent vecteurs d'un délaissement accru ("Ne t'inquiète pas pour Mamie, elle a le robot pour lui tenir compagnie. ») : il préconise donc l'usage de robots-outils pour le personnel médical et non de robotssoignants pour les seniors. Monique Lanoix insiste quant à elle sur le fait que le recours au robot-phoque PARO en accompagnement gériatrique peut augmenter l'isolement institutionnel du personnel aussi bien que des patients. Délaissant le terrain purement humain pour s'intéresser à la traite laitière, Sandra Blouin montre que l'absence de réciprocité dans la relation robotique met à mal le lien entre l'éleveur et son troupeau, accroissant potentiellement le mal-être animal. Georges Chapouthier met en évidence le fossé empathique qui sépare animaux et robots, et montre que le vécu émotionnel et le ressenti de la douleur, partagé par les humains et les animaux, peuvent former la base d'une morale commune, inaccessible aux robots. Les deux derniers chapitres utilisent le robot pour questionner notre rapport à la biosphère : Louis-Étienne Pigeon analyse la robotisation des corps et des milieux et Catherine Larrère interroge la place des robots dans la transition écologique.

6 Comparativement aux hypothèses prospectives de la première partie, ce second temps de l'ouvrage ramène les questionnements à un niveau socio-économique, technique, psychologique et politique bienvenu pour dégonfler certaines anticipations outrancières. Les grandes interrogations sur le statut moral et ontologique des robots apparaissent un peu déplacées quand on découvre qu'un simple robot-phoque peut accroître la vulnérabilité émotionnelle d'une vieille dame. On ne peut bien sûr blâmer les deux auteurs ayant très sérieusement coordonné l'ensemble de l'ouvrage quant au fait que les discours sur la robotique soient saturés de prophéties anticipatrices, même s'il aurait sans doute été possible de réduire le nombre de textes du volume pour se recentrer sur les études de terrain mobilisées dans la seconde partie.

7 Enfin, on peut regretter que la lecture de l'ouvrage laisse entrevoir une liste de sujets que l'on aurait aimé voir traités: racines de la mythologie d'une robotique qui supplanterait l'humain, sociologie de la Silicon Valley et de ses "influenceurs" mondiaux, travail concret des petites mains du numérique ${ }^{2}$, réalités matérielles d'où émergent les automates informatiques bien réels d'aujourd'hui - spéculation boursière en temps réel, fibre optique, terminaux, datacenters, etc. La liste n'étant pas close, on referme l'ouvrage avec perplexité, dubitatif quant au temps académique consacré à des sujets qui ne deviendront peut-être jamais réalité, alors que d'autres, structurants pour le monde d'aujourd'hui, sont parfois délaissés par la recherche contemporaine.

\section{BIBLIOGRAPHIE}

CARNINO Guillaume et MARQUET Clément, « Du mythe de l'automatisation au savoir-faire des petites mains : une histoire des datacenters par la panne », Artefact. Techniques, histoire et sciences humaines, 2019, n 11, p. 161-188. 
DENIS Jérôme et PONTILLE David (dir.), Les petites mains de la société de l'information, Revue d'anthropologie des connaissances, 2012, nº 6(1).

\section{NOTES}

1. Voir par exemple Carnino et Marquet, 2019.

2. Denis et Pontille, 2012.

\section{AUTEURS}

GUILLAUME CARNINO

UTC, EA COSTECH 